Applied Mathematical Sciences, Vol. 7, 2013, no. 13, 645 - 650

\title{
Longest Common Parameterized Subsequences with Fixed Common Substring
}

\author{
Anna Gorbenko \\ Department of Intelligent Systems and Robotics \\ Ural Federal University \\ 620083 Ekaterinburg, Russia \\ gorbenko.ann@gmail.com \\ Vladimir Popov \\ Department of Intelligent Systems and Robotics \\ Ural Federal University \\ 620083 Ekaterinburg, Russia \\ Vladimir.Popov@usu.ru
}

\begin{abstract}
In this paper we consider the problem of the longest common parameterized subsequence with fixed common substring (STR-IC-LCPS). In particular, we show that STR-IC-LCPS is NP-complete. We describe an approach to solve STR-IC-LCPS. This approach is based on an explicit reduction from the problem to the satisfiability problem.
\end{abstract}

Keywords: parameterized pattern matching, satisfiability, NP-complete

Different variants of the problem of the longest common subsequence are extensively used as distance measures for strings. In particular, the following problem was proposed in [1] (see also [2]). STR-IC-LCS:

Given two strings $S_{1}$ and $S_{2}$ and a constraint pattern $P$ of length $n, m$, and $r$, respectively, find a longest common subsequence of $S_{1}$ and $S_{2}$ including $P$ as a substring.

Another well-studied string comparison measure is that of parameterized matching (basic definitions and results can be found in [3]). It is natural to attempt to accommodate parameterized matching along with some other 
distance measures. In this paper we consider a parameterized variant of STRIC-LCS.

THE PROBLEM OF THE LONGEST COMMON PARAMETERIZED SUBSEQUENCE WITH FIXED COMMON SUBSTRING (STR-IC-LCPS):

Instance: An alphabet $\Sigma \cup \Pi$, sequences $S_{1}$ and $S_{2}$ over $\Sigma \cup \Pi$, a string $P$ over $\Sigma$, and positive integer $k$.

Question: Is there a sequence $T,|T| \geq k$, such that $P$ is a substring of $T$ and $T$ is a parameterized subsequence of $S_{1}$ and $S_{2}$ ?

It is clear that there is some connection between longest common parameterized subsequences and longest common parameterized subsequences with fixed common substring. In particular, if $T_{1}$ is a longest common parameterized subsequence of $S_{1}$ and $S_{2}$ and $T_{2}$ is a parameterized subsequence of $S_{1}$ and $S_{2}$ with fixed common substring $P$, then $\left|T_{1}\right| \geq\left|T_{2}\right|$. However, $T_{1}$ and $T_{2}$ may significantly differ from each other.

Theorem 1. For any $n$ and $k$, there are sequences $S_{1}, S_{2}, P, T_{1}$, and $T_{2}$ such that

(1) $T_{1}$ is a longest common parameterized subsequence of $S_{1}$ and $S_{2}$;

(2) $T_{2}$ is a longest common parameterized subsequence of $S_{1}$ and $S_{2}$ with fixed common substring $P$;

(3) $\left|T_{2}\right| \geq n$;

(4) $\left|T_{1}\right| \geq\left|T_{2}\right|+k$.

Proof. Let $\Sigma=\{a, b\}, \Pi=\emptyset, S_{1}=a^{s} b^{t}, S_{2}=b^{t} a^{s}, P=b^{t}$. We assume that $s>t+k$ and $t>n$. Let $T_{1}=a^{s}$. Since $s>t+k$, it is clear that $T_{1}$ is a longest common parameterized subsequence of $S_{1}$ and $S_{2}$. Let $T_{2}=b^{t}$. It is easy to see that $T_{2}$ is a parameterized subsequence of $S_{1}$ and $S_{2}$. Since $P=b^{t}$, it is clear that $P$ is a substring of $T_{2}$. In view of $P=b^{t}$, it is easy to check that $T_{2}$ is a longest common parameterized subsequence of $S_{1}$ and $S_{2}$ with fixed common substring $P$. By definition of $T_{2}$, in view of $t>n$, it is clear that $\left|T_{2}\right| \geq n$. Since $s>t+k$, it is easy to see that $\left|T_{1}\right| \geq\left|T_{2}\right|+k$.

Now we consider the complexity of STR-IC-LCPS.

Theorem 2. STR-IC-LCPS is NP-complete.

Proof. It is clear that STR-IC-LCPS is in NP. In order to prove that STR-IC-LCPS is NP-hard, we shall reduce LCPS (see [4]) to STR-ICLCPS.

LCPS:

InSTANCE: An alphabet $\Sigma \cup \Pi$, sequences $S_{1}$ and $S_{2}$ over $\Sigma \cup \Pi$, and positive integer $k$.

QUESTION: Is there a sequence $T,|T| \geq k$, that is a parameterized subsequence of $S_{1}$ and $S_{2}$ ?

Let $\Sigma \cup \Pi$ be an alphabet. Let $S_{1}$ and $S_{2}$ are sequences over $\Sigma \cup \Pi$.

We assume that $c$ is a letter such that $c \notin \Sigma \cup \Pi$. Let $\Sigma^{\prime}=\Sigma \cup\{c\}$. Let $P=c$ and $S_{i}^{\prime}=c S_{i}, i \in\{1,2\}$. 
It is easy to check that $T$ is a longest common parameterized subsequence of $S_{1}$ and $S_{2}$ if and only if $c T$ is a longest common parameterized subsequence of $S_{1}^{\prime}$ and $S_{2}^{\prime}$ with fixed common substring $P$. Note that LCPS is NP-complete [4]. Therefore, STR-IC-LCPS is NP-complete.

Encoding different hard problems as Boolean satisfiability and solving them with very efficient satisfiability algorithms has caused considerable interest (see e.g. [5] - [22]). We consider an explicit reduction from STR-IC-LCPS to the satisfiability problem.

Let $\Sigma=\left\{a_{1}, a_{2}, \ldots, a_{|\Sigma|}\right\}, \Pi=\left\{b_{1}, b_{2}, \ldots, b_{|\Pi|}\right\}$,

$$
\begin{aligned}
& \varphi[1]=\wedge_{1 \leq i \leq k} \vee_{1 \leq j \leq|\Sigma \cup \Pi|} x[i, j] \\
& \varphi[2]=\wedge_{1 \leq i \leq k} \wedge_{1 \leq j[1]<j[2] \leq|\Sigma \cup \Pi|}(\neg x[i, j[1]] \vee \neg x[i, j[2]]), \\
& \varphi[3]=\wedge_{1 \leq i \leq|P|} \vee_{1 \leq j \leq|\Sigma|} u[i, j], \\
& \varphi[4]=\wedge_{1 \leq i \leq|P|} \wedge_{1 \leq j[1]<j[2] \leq|\Sigma|}(\neg u[i, j[1]] \vee \neg u[i, j[2]]), \\
& \varphi[5]=\wedge_{1 \leq i \leq|P|} \wedge_{1 \leq j \leq|\Sigma|, P[i] \neq a_{j}} \neg u[i, j], \\
& \varphi[6]=\vee_{1 \leq i \leq k-|P|+1} v[i], \\
& \varphi[7]=\wedge_{1 \leq i \leq k-|P|+1,1 \leq j \leq|P|, 1 \leq s \leq|\Sigma|}((\neg v[i] \vee \neg u[j, s] \vee x[j+i-1, s]) \wedge \\
& (\neg v[i] \vee u[j, s] \vee \neg x[j+i-1, s])), \\
& \varphi[8]=\wedge_{1 \leq i \leq\left|S_{2}\right|} \vee_{1 \leq j \leq|\Sigma \cup \Pi|} y[i, j], \\
& \varphi[9]=\wedge_{1 \leq i \leq\left|S_{2}\right|} \wedge_{1 \leq j[1]<j[2] \leq|\Sigma \cup \Pi|}(\neg y[i, j[1]] \vee \neg y[i, j[2]]), \\
& \varphi[10]=\wedge_{1 \leq i \leq\left|S_{2}\right|, S_{2}[i] \in \Pi} \wedge_{1 \leq j \leq|\Sigma|} \neg y[i, j], \\
& \varphi[11]=\wedge_{1 \leq i[1]<i[2] \leq\left|S_{2}\right|},((\neg y[i[1], j] \vee y[i[2], j]) \wedge(y[i[1], j] \vee \neg y[i[2], j])), \\
& S_{2}[i[1]]=S_{2}[i[2]] \text {, } \\
& S_{2}[i[2]] \in \Pi \text {, } \\
& 1 \leq j \leq|\Sigma \cup \Pi|
\end{aligned}
$$

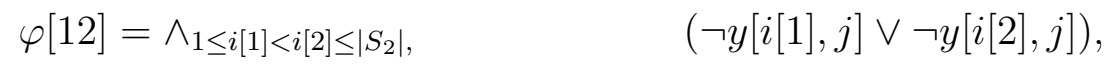

$$
\begin{aligned}
& S_{2}[i[1]] \neq S_{2}[i[2]], S_{2}[i[1]] \in \Pi, S_{2}[i[2]] \in \Pi, \\
& 1 \leq j \leq|\Sigma \cup \Pi| \\
& \varphi[13]=\wedge_{1 \leq i \leq\left|S_{1}\right|, 1 \leq j \leq k, 1 \leq l \leq|\Sigma \cup \Pi|, S_{1}[i] \neq a_{l}, S_{1}[i] \neq b_{l-|\Sigma|}}(\neg z[1, i, j] \vee \neg x[j, l]), \\
& \varphi[14]=\wedge_{1 \leq i \leq\left|S_{2}\right|, 1 \leq j \leq k, 1 \leq l \leq|\Sigma \cup \Pi|, S_{2}[i] \neq a_{l}, S_{2}[i] \in \Sigma}(\neg z[2, i, j] \vee \neg x[j, l]),
\end{aligned}
$$

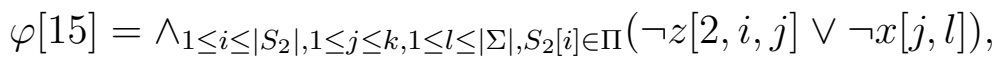

$$
\begin{aligned}
& \varphi[16]=\wedge_{1 \leq i \leq\left|S_{2}\right|, 1 \leq j \leq k,|\Sigma|+1 \leq l \leq|\Sigma \cup \Pi|, S_{2}[i] \in \Pi}((\neg z[2, i, j] \vee \neg y[i, l] \vee x[j, l]) \wedge \\
& (\neg z[2, i, j] \vee y[i, l] \vee \neg x[j, l])), \\
& \varphi[17]=\wedge_{1 \leq i \leq 2,1 \leq j \leq\left|S_{i}\right|, 1 \leq l[1]<l[2] \leq k}(\neg z[i, j, l[1]] \vee \neg z[i, j, l[2]]),
\end{aligned}
$$




$$
\begin{gathered}
\varphi[18]=\wedge_{1 \leq i \leq 2,1 \leq l \leq k} \vee_{1 \leq j \leq\left|S_{i}\right|} z[i, j, l], \\
\varphi[19]=\wedge_{1 \leq i \leq 2,1 \leq j[1] \leq\left|S_{i}\right|,(\neg z[i, j[1], l[1]] \vee \neg z[i, j[2], l[2]]),} \\
\begin{array}{c}
1 \leq l[1] \leq k, \\
j[2]>j[1], l[2]<l[1]
\end{array} \\
\xi=\wedge_{i=1}^{19} \varphi[i] .
\end{gathered}
$$

It is easy to check that there is a sequence $T,|T| \geq k$, such that $P$ is a substring of $T$ and $T$ is a parameterized subsequence of $S_{1}$ and $S_{2}$ if and only if $\xi$ is satisfiable. It is clear that $\xi$ is a CNF. So, $\xi$ gives us an explicit reduction from STR-IC-LCPS to SAT. Now, using standard transformations (see e.g. [23]) we can obtain an explicit transformation $\xi$ into $\zeta$ such that $\xi \Leftrightarrow \zeta$ and $\zeta$ is a 3-CNF. Clearly, $\zeta$ gives us an explicit reduction from STR-IC-LCPS to 3SAT.

We have designed generators of natural random instances for STR-ICLCPS. We have consider our genetic algorithms OA[1] (see [24]), OA[2] (see [25]), OA[3] (see [26]), and OA[4] (see [27]) for SAT. We have used heterogeneous cluster. Each test was runned on a cluster of at least 100 nodes. Note that due to restrictions on computation time (20 hours) we used savepoints. Selected experimental results are given in Table 1.

\begin{tabular}{|l|lll|}
\hline time & average & $\max$ & best \\
\hline $\mathrm{OA}[1]$ & $2.7 \mathrm{~h}$ & $31.72 \mathrm{~h}$ & $14 \mathrm{~min}$ \\
$\mathrm{OA}[2]$ & $2.32 \mathrm{~h}$ & $26.4 \mathrm{~h}$ & $18 \mathrm{~min}$ \\
$\mathrm{OA}[3]$ & $1.74 \mathrm{~h}$ & $29 \mathrm{~h}$ & $26 \mathrm{~min}$ \\
$\mathrm{OA}[4]$ & $1.96 \mathrm{~h}$ & $14.4 \mathrm{~h}$ & $21.2 \mathrm{~min}$ \\
\hline
\end{tabular}

Table 1: Experimental results for STR-IC-LCPS.

ACKNOWLEDGEMENTS. The work was partially supported by Analytical Departmental Program "Developing the scientific potential of high school" 8.1616.2011.

\section{References}

[1] Y.-C. Chen and K.-M. Chao, On the generaized constrained longest common subsequence problem, Journal of Combinatorial Optimization, 21 (2011), 383-392.

[2] E. Farhana, J. Ferdous, T. Moosa, and M.S. Rahman, Finite Automata Based Algorithms for the Generalized Constrained Longest Common Subsequence Problems, Lecture Notes in Computer Science, 6393 (2010), 243249. 
[3] A. Gorbenko and V. Popov, The Longest Common Parameterized Subsequence Problem, Applied Mathematical Sciences, 6 (2012), 2851-2855.

[4] O. Keller, T. Kopelowitz, and M. Lewenstein, On the Longest Common Parameterized Subsequence, Theoretical Computer Science, 410 (2009), 5347-5353.

[5] A. Gorbenko and V. Popov, On the Problem of Sensor Placement, Advanced Studies in Theoretical Physics, 6 (2012), 1117-1120.

[6] A. Gorbenko and V. Popov, On the Longest Common Subsequence Problem, Applied Mathematical Sciences, 6 (2012), 5781-5787.

[7] A. Gorbenko and V. Popov, The Binary Paint Shop Problem, Applied Mathematical Sciences, 6 (2012), 4733-4735.

[8] A. Gorbenko, M. Mornev, V. Popov, and A. Sheka, The Problem of Sensor Placement, Advanced Studies in Theoretical Physics, 6 (2012), 965-967.

[9] A. Gorbenko, V. Popov, and A. Sheka, Localization on Discrete Grid Graphs, Lecture Notes in Electrical Engineering, 107 (2012), 971-978.

[10] A. Gorbenko and V. Popov, The Problem of Selection of a Minimal Set of Visual Landmarks, Applied Mathematical Sciences, 6 (2012), 4729-4732.

[11] A. Gorbenko and V. Popov, The Longest Common Parameterized Subsequence Problem, Applied Mathematical Sciences, 6 (2012), 2851-2855.

[12] A. Gorbenko and V. Popov, Programming for Modular Reconfigurable Robots, Programming and Computer Software, 38 (2012), 13-23.

[13] A. Gorbenko, M. Mornev, V. Popov, and A. Sheka, The problem of sensor placement for triangulation-based localisation, International Journal of Automation and Control, 5 (2011), 245-253.

[14] A. Gorbenko, M. Mornev, and V. Popov, Planning a Typical Working Day for Indoor Service Robots, IAENG International Journal of Computer Science, 38 (2011), 176-182.

[15] A. Gorbenko and V. Popov, The set of parameterized k-covers problem, Theoretical Computer Science, 423 (2012), 19-24.

[16] A. Gorbenko and V. Popov, Clustering Algorithm in Mobile Ad Hoc Networks, Advanced Studies in Theoretical Physics, 6 (2012), 1239-1242.

[17] A. Gorbenko and V. Popov, The Problem of Finding Two Edge-Disjoint Hamiltonian Cycles, Applied Mathematical Sciences, 6 (2012), 6563-6566. 
[18] A. Gorbenko and V. Popov, Hamiltonian Alternating Cycles with Fixed Number of Color Appearances, Applied Mathematical Sciences, 6 (2012), 6729-6731.

[19] A. Gorbenko and V. Popov, Footstep Planning for Humanoid Robots, Applied Mathematical Sciences, 6 (2012), 6567-6571.

[20] A. Gorbenko and V. Popov, Multiple Occurrences Shortest Common Superstring Problem, Applied Mathematical Sciences, 6 (2012), 6573-6576.

[21] A. Gorbenko and V. Popov, The Far From Most String Problem, Applied Mathematical Sciences, 6 (2012), 6719-6724.

[22] A. Gorbenko and V. Popov, Multi-agent Path Planning, Applied Mathematical Sciences, 6 (2012), 6733-6737.

[23] A. Gorbenko and V. Popov, The c-Fragment Longest Arc-Preserving Common Subsequence Problem, IAENG International Journal of Computer Science, 39 (2012), 231-238.

[24] A. Gorbenko and V. Popov, On the Problem of Placement of Visual Landmarks, Applied Mathematical Sciences, 6 (2012), 689-696.

[25] A. Gorbenko and V. Popov, Computational Experiments for the Problem of Selection of a Minimal Set of Visual Landmarks, Applied Mathematical Sciences, 6 (2012), 5775-5780.

[26] A. Gorbenko and V. Popov, Task-resource Scheduling Problem, International Journal of Automation and Computing, 9 (2012), 429-441.

[27] A. Gorbenko and V. Popov, SAT Solvers for the Problem of Sensor Placement, Advanced Studies in Theoretical Physics, 6 (2012), 1235-1238.

\section{Received: November 1, 2012}

